\title{
Anti-inflammatory activity of liposomes of Asparagus racemosus root extracts prepared by various methods
}

\author{
NATHSIREE PLANGSOMBAT ${ }^{1}$, KANIN RUNGSARDTHONG $^{2}$, LALANA KONGKANERAMIT ${ }^{3}$, \\ NETI WARANUCH ${ }^{4}$ and NARONG SARISUTA ${ }^{1,5}$
}

\author{
${ }^{1}$ Department of Manufacturing Pharmacy, Faculty of Pharmacy, Mahidol University, Bangkok 10400; \\ ${ }^{2}$ Department of Pharmaceutical Care, Faculty of Pharmacy, Thammasat University, Rangsit Center, Khlong Luang, \\ Pathumthani 12121; ${ }^{3}$ Department of Pharmaceutical Technology, Faculty of Pharmacy, Srinakharinwirot University, \\ Ongkharak, Nakhonnayok 26120; ${ }^{4}$ Department of Pharmaceutical Technology, Faculty of Pharmaceutical Sciences, \\ Naresuan University, Phitsanulok, Phitsanulok 65000; ${ }^{5}$ Department of Pharmaceutical Sciences, \\ Faculty of Pharmacy, Thammasat University, Rangsit Center, Khlong Luang, Pathumthani 12121, Thailand
}

Received June 18, 2015; Accepted July 29, 2016

DOI: $10.3892 / \mathrm{etm} .2016 .3661$

\begin{abstract}
Asparagus racemosus root extracts (AR) have been reported to possess a variety of pharmacological properties. The aim of the present study was to develop liposomes of AR and to assess their physicochemical characteristics and anti-inflammatory activity in the monocytic leukemia cell line THP-1. Liposomes containing various ratios of AR to lipid and a phosphatidylcholine to cholesterol molar ratio of 7:3 were prepared by thin-film hydration (TF), reverse-phase evaporation (REV) and polyol dilution (PD). The results showed that AR liposomes prepared by TF had a multilamellar structure and a large size, whereas those prepared by REV and PD were oligolamellar in structure, and of a smaller size. The particle sizes and zeta potentials of the liposomes ranged from 196.5 to $456.6 \mathrm{~nm}$ and from -4.34 to $-18.94 \mathrm{mV}$, respectively. The AR to lipid ratio was shown to have no significant influence on particle size, while the zeta potential generally increased with increasing AR to lipid ratio. The highest entrapment efficiency values were detected in liposomes with an AR to lipid ratio of 1:5, and for liposomes prepared by TF, REV and PD methods, the entrapment efficiencies were 55.71 $\pm 2.04,56.21 \pm 3.59$ and $67.68 \pm 1.37 \%$, respectively. AR was found to exert no toxicity on THP-1 cells. The maximum anti-inflammatory activities of AR and AR liposomes, evaluated in terms of the percentage inhibition of tumor necrosis factor- $\alpha$ in THP-1 cells, were
\end{abstract}

Correspondence to: Dr Kanin Rungsardthong, Department of Pharmaceutical Care, Faculty of Pharmacy, Thammasat University, Rangsit Center, 99 Moo 18 Phahonyothin Road, Khlong Luang, Pathumthani 12121, Thailand

E-mail: kanin_rungsardthong@yahoo.com

Key words: Asparagus racemosus, liposomes, thin-film hydration, reverse-phase evaporation, polyol dilution, anti-inflammatory activity, cytotoxicity $\sim 52 \%$ at a concentration of $1 \mu \mathrm{g} / \mathrm{ml}$. It can be concluded from the present study that AR liposomes have the potential to be used a formulation for topical and/or transdermal drug delivery to provide anti-inflammatory activity.

\section{Introduction}

Asparagus racemosus belongs to the family Liliaceae, and is commonly known as Satavari. In Thai, it is termed Rak-Sam-Sib or Sam-Roi-Rak. The plant is frequently found at low altitudes in shaded areas and in tropical climates throughout Asia, Australia and Africa. Steroidal saponins (such as shatavarin I-IV) are the major constituents of the plant. The pharmacological activities of A. racemosus root extracts (AR) have been reported, including antiulcer, antioxidant, immunomodulatory, antidiabetic, antidiarrhoeal, phytoestrogenic, anti-aging and adaptogenic properties. Root of A. racemosus can be used as a tonic, and is considered to be bittersweet with emollient, cooling, nervine, constipating, galactagogic, aphrodisiac, diuretic, rejuvenating, carminative, stomachic and antiseptic properties. The root of A. racemosus has several beneficial effects that are suggested for the treatment of nervous disorders, dyspepsia, diarrhea, tumors and inflammation (1-3).

In general, liposomes are microscopic spherical vesicles consisting of one or more lipid bilayers with an aqueous phase inside the vesicle and between the lipid bilayers, and are most commonly composed of phospholipids encapsulating a volume of aqueous medium (4). Liposomes contain a hydrophilic compartment between the bilayers and a lipophilic compartment within the bilayer membranes. As a result, water-soluble drugs are able to localize in hydrophilic compartments, while lipid soluble and amphiphilic drugs insert themselves into the bilayer membranes (5). The potential applications of liposomes as targeted drug delivery systems are acknowledged for a variety of drugs such as therapeutic peptides and proteins, diagnostic agents, drugs of small molecular weight, hormones, enzymes, antibiotics, antifungal and anticancer agents as well as macromolecules (such as herbal extracts) (6). In addition, liposomes 
have several beneficial effects when used in combination with herbal drugs, including increased solubility, enhanced bioavailability, reduced toxicity and increased pharmacological activity of the drug, in addition to promotion of its sustainability and protection against physical and chemical degradation (7).

The aim of the current study was to prepare liposomes of A. racemosus root extracts by thin-film hydration (TF), reverse-phase evaporation (REV) and polyol dilution (PD) methods with various extract to lipid ratios. The physicochemical properties of the liposomes, including morphology, size, surface charge and drug entrapment, in addition to their in vitro anti-inflammatory properties, were subsequently evaluated.

\section{Materials and methods}

Materials. A. racemosus root extracts (AR) were obtained according to an extraction procedure described previously (8). Phosphatidylcholine (PC; Phospholipon 90G) was donated by Rhône Poulenc Rorer (Köln, Germany). Cholesterol (CHOL) was purchased from Carlo Erba Reagents Srl (Rodano, Italy). Lipopolysaccharide (LPS) from $E$. coli $026: \mathrm{B}_{6}$ and vanillin were purchased from Sigma-Aldrich (Merck Millipore; Darmstadt, Germany). Chloroform, methanol, dichloromethane and sulfuric acid 98\% (v/v) were from Labscan, Ltd., Co. (Dublin, Ireland). Dibasic potassium phosphate, monobasic potassium phosphate, triethanolamine and sodium hydroxide were purchased from Ajax Finechem (Auckland, New Zealand). Absolute ethanol and propylene glycol were from Merck KGaA (Darmstadt, Germany) and Dow Chemical (Midland, MI, USA), respectively. Dulbecco's phosphate buffered saline (PBS), 3-(4,5-dimethyl-2-thiazolyl)-2,5-diphenyl-2H-tetrazolium bromide (MTT), RPMI-1640 medium, and penicillin/streptomycin were purchased from Gibco (Thermo Fisher Scientific, Inc., Waltham, MA, USA). Fetal bovine serum (FBS) was from Hyclone (GE Healthcare Life Sciences, Logan, UT, USA). A tumor necrosis factor (TNF)- $\alpha$ enzymelinked immunosorbent assay (ELISA) kit was purchased from BD Biosciences (San Jose, CA, USA). All other chemicals were of analytical grades.

\section{Preparation of AR liposomes by various methods}

TF method. Total lipid mixture (100 mg) with a PC to CHOL molar ratio of 7:3 was dissolved in $20 \mathrm{ml}$ chloroform in a $250-\mathrm{ml}$ round bottom flask. The solvent was removed from the lipid phase by rotary evaporator (Eyela A3S; Tokyo Rikakikai Co., Ltd., Tokyo, Japan) at $35^{\circ} \mathrm{C}$ under reduced pressure until a dry thin film was deposited on the wall of flask. The dry thin film was hydrated with an aqueous phase containing various ratios by weight of $A R$ to total lipid at 0:5, 1:5, 2:5 and 3:5 in $20 \mathrm{ml}$ isotonic phosphate buffer $\mathrm{pH} 7.0$ at above phase transition temperature in order to obtain a homogeneous milky white suspension of liposomes. The suspension was allowed to stand for further $2 \mathrm{~h}$ at room temperature to achieve complete swelling of the lipid film. The unentrapped AR was separated using ultracentrifugation (Optima Max-XP; Beckman Coulter, Inc., Brea, CA, USA ) at 236,400 x g for $1 \mathrm{~h}$ at $4^{\circ} \mathrm{C}$. Liposomes were washed several times with isotonic phosphate buffer $\mathrm{pH} 7.0$ until the concentration of AR in the supernatant was $<1.0 \%$. The purified liposomes were transferred to a container and stored at $4^{\circ} \mathrm{C}$ prior to use.
REV method. The total lipid mixture (100 mg) with a PC to CHOL molar ratio of 7:3 was dissolved in $60 \mathrm{ml}$ dichloromethane in a $250-\mathrm{ml}$ round bottom flask, after which the aqueous phase containing various ratios by weight of AR to total lipid $(0: 5,1: 5,2: 5$ and $3: 5 \mathrm{in} 20 \mathrm{ml}$ isotonic phosphate buffer, $\mathrm{pH}$ 7.0) was introduced into the lipid solution through a 22-gauge hypodermic needle with a $5-\mathrm{ml}$ syringe. The flask was immediately sealed with a glass stopper and placed in an ultrasonic bath (Bransonic 2510; Branson, Danbury, CT, USA). The mixture was sonicated at $7^{\circ} \mathrm{C}$ for $10 \mathrm{~min}$ to form an water-in-oil (w/o) emulsion. The emulsion was then transferred to a rotary evaporator to gradually evaporate the solvent under reduced pressure at $35^{\circ} \mathrm{C}$ until a viscous gel was formed, indicating phase reversion into oil-in-water $(\mathrm{o} / \mathrm{w})$ emulsion. Continuation of evaporation eventually resulted in a homogeneous aqueous dispersion. Subsequently, liposomal dispersions were subjected to complete removal of remnant traces of organic solvent in a rotary evaporator for $\geq 30 \mathrm{~min}$ at 35 to $37^{\circ} \mathrm{C}$. Finally, the obtained liposomal dispersions were purified by ultracentrifugation in a similar manner as previously described in the TF method, and stored at $4^{\circ} \mathrm{C}$ until further use.

PD method. Total lipid mixture (100 mg) with a PC to CHOL molar ratio of 7:3 was dissolved in $4 \mathrm{ml}$ propylene glycol at $50^{\circ} \mathrm{C}$. The aqueous phase containing various ratios by weight of AR to total lipid (0:5, 1:5, 2:5 and 3:5) in $16 \mathrm{ml}$ isotonic phosphate buffer ( $\mathrm{pH} 7.0$ ) was preheated to $50^{\circ} \mathrm{C}$ and gradually injected into the lipid solution, and then mixed for $45 \mathrm{~min}$. The obtained liposomal dispersions were subsequently purified by ultracentrifugation in a similar manner as previously described in the TF method, and stored at $4^{\circ} \mathrm{C}$ until required.

\section{Physicochemical characterization of AR liposomes}

Morphology. The microscopic appearance of prepared AR liposomes was examined using a negative-staining transmission electron microscope (Tecnai $G^{2} 20$; Fei Company, Eindhoven, The Netherlands). Briefly, a drop of liposomal dispersion was placed on a 300-mesh copper grid. After the liposomes had adsorbed onto the grid, the excess liquid was removed using filter paper. The sample that remained on the grid was stained with a $2 \%$ aqueous solution of uranyl acetate and allowed to air dry for $\sim 30 \mathrm{~min}$. Subsequently, the sample was stored in a desiccator prior to use.

Particle size. Particle sizes and size distribution were determined by photon correlation spectroscopy (PCS) using dynamic light scattering (Zetasizer Nano ZS; Malvern Instruments, Ltd., Malvern, UK) at a temperature of $25^{\circ} \mathrm{C}$. The samples (liposomal dispersions) were diluted with an isotonic phosphate buffer $\mathrm{pH} 7.0$ until their concentrations were low enough to avoid hydrodynamic and electrostatic interactions between vesicles, yet high enough to be reliable in autocorrelation functions from PCS measurement. The measurements were performed in triplicate and expressed as a $\mathrm{z}$-average in addition to the polydispersity index, which indicates the size distribution of the liposomes.

Zeta potential. Liposomal dispersions were diluted with an appropriate buffer solution, after which the zeta potentials were determined by laser Doppler electrophoretic mobility measurements using the Zetasizer Nano ZS.

Entrapment efficiency. The entrapment efficiencies of AR liposomes prepared by various methods were determined by 
ultracentrifugation. The liposomal dispersions were centrifuged at $236,400 \times \mathrm{g}$ for $1 \mathrm{~h}$ at $4^{\circ} \mathrm{C}$. The $\mathrm{AR}$ liposomes were lysed with methanol and measured spectrophotometrically at a wavelength of $544 \mathrm{~nm}$ (UV-2600; Shimadzu Corporation, Tokyo, Japan) using a quantitative test for total saponins (8). All experiments were performed in triplicate. The entrapment efficiency and loading capacity of AR liposomes were calculated using the following equations, respectively.

$$
\begin{aligned}
& \text { Entrapment efficiency }(\%)=\frac{\text { Amount of drug entrapped in liposomes }}{\text { Total amount of drug added in liposomes }} \times 100 \\
& \text { Loading capacity }(\%)=\frac{\text { Amount of drug entrapped in liposomes }}{\text { Total amount of lipid in liposomes }} \times 100
\end{aligned}
$$

Cytotoxicity test. Human leukemia monocytic (THP-1) cells (American Type Culture Collection, Manassas, VA, USA) were used in an MTT assay. The cells were cultured in RPMI-1640 media containing 10\% FBS and supplemented with $100 \mathrm{U} / \mathrm{ml}$ penicillin and $100 \mu \mathrm{g} / \mathrm{ml}$ streptomycin. The cell culture was maintained at $37^{\circ} \mathrm{C}$ in a humidified $5 \% \mathrm{CO}_{2}$ atmosphere (BINDER GmbH, Tuttlingen, Germany). THP-1 cells were seeded in 96-well plate (Corning Costar, New York, NY, USA) at $2.5 \times 10^{4}$ cells/well and induced by $100 \mathrm{nM}$ phorbolmyristate acetate (PMA) for $48 \mathrm{~h}$ to allow the cells to differentiate into macrophages. Undifferentiated monocytic cells were removed by washing with culture medium and fresh medium without any FBS or PMA additive was added. The cells were then incubated for an additional $24 \mathrm{~h}$ to allow the effects of PMA upon cell activation to abate. Subsequently, the medium was removed and replaced by various concentrations of $\operatorname{AR}(1,10,100,500$ and 1,000 $\mu \mathrm{g} / \mathrm{ml})$, AR liposomes (5, $10,50,100$ and $100 \mu \mathrm{g} / \mathrm{ml})$ and medium alone. After $24 \mathrm{~h}$ of incubation, the cells in each well were washed with PBS and $100 \mu \mathrm{l}$ of $0.5 \mathrm{mg} / \mathrm{ml}$ MTT solution was added. The cells were then incubated for a further $4 \mathrm{~h}$ at $37^{\circ} \mathrm{C}$ with $5 \% \mathrm{CO}_{2}$, after which the MTT solution in each well was carefully discarded and replaced by $50 \mu \mathrm{l}$ isopropanol to dissolve formazan crystals. The absorbance of the formazan solution was measured at $590 \mathrm{~nm}$. The cell viability was calculated using the following equation.

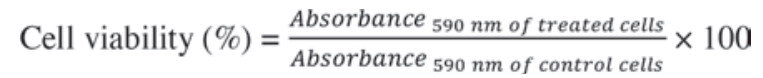

\section{In vitro anti-inflammatory activity}

The in vitro anti-inflammatory activity was assessed in terms of inhibition of TNF- $\alpha$ in LPS-activated THP-1 monocytic cells. TNF- $\alpha$ is an important pro-inflammatory cytokine produced by monocytes and macrophages during inflammatory responses. In the present study, the secreted TNF- $\alpha$ levels in the supernatant of the THP-1 cell culture were measured using a TNF- $\alpha$ ELISA kit (BD OptEIA; BD Biosciences) with reference to a TNF- $\alpha$ calibration curve.

Cell culture. THP-1 cells were grown in RPMI-1640 media containing $10 \%$ FBS supplemented with $100 \mathrm{U} / \mathrm{ml}$ penicillin and $100 \mu \mathrm{g} / \mathrm{ml}$ streptomycin. The cell culture was maintained at $37^{\circ} \mathrm{C}$ in humidified conditions with $5 \%$ atmospheric $\mathrm{CO}_{2}$. The cell suspension was prepared in RPMI-1640 medium at $2.5 \times 10^{5}$ cells $/ \mathrm{ml}$ and preactivated with $100 \mathrm{nM}$ PMA in a 48-well plate for $48 \mathrm{~h}$ in order to transform into macrophages. After incubation, undifferentiated monocytic cells were removed by washing with culture medium and fresh medium (without PMA) was added. The cells were then incubated for an additional $24 \mathrm{~h}$ to allow the effects of PMA upon cell activation to abate. Subsequently, the medium was removed and the cells were treated with various concentrations of AR and AR liposomes. Dexamethasone $(1 \mu \mathrm{M})$ and vehicle were used as a reference drug and control, respectively. After 30 min pretreatment with the test samples, the cells were further stimulated with $0.1 \mu \mathrm{g} / \mathrm{ml}$ LPS for $24 \mathrm{~h}$. Following incubation, cell-free supernatants were collected and TNF- $\alpha$ concentration levels were determined using a TNF- $\alpha$ ELISA kit.

Determination of secreted TNF- $\alpha$ in culture supernatants. The TNF- $\alpha$ concentrations in the supernatants were determined using a TNF- $\alpha$ ELISA kit (BD OptEIA) according to the manufacturer's instructions. Briefly, 96-well ELISA plates were coated with the monoclonal antibody specific for TNF. The standard and samples were added to the wells, and any TNF present was bound to the immobilized antibody. The wells were washed and a streptavidin-horseradish peroxidase conjugate mixed with a biotinylated anti-human TNF antibody was added, producing an antibody-antigen-antibody 'sandwich'. The wells were then washed and a 3,3',5,5'-tetramethylbenzidine substrate solution was added and a blue color was generated, which was in direct proportion to the amount of TNF present in the initial sample. The stop solution changed the color from blue to yellow, and the microwell absorbance was read at $450 \mathrm{~nm}$ with a multiwell plate reader (FLUOstar OPTIMA; BMG Labtech, Durham, NC, USA). The percentage of TNF- $\alpha$ inhibition was calculated using the following equation.

$$
\text { TNF- } \alpha \text { inhibition }(\%)=\left[\frac{T N F_{\text {negative control }}-T N F_{\text {sample }}}{T N F_{\text {negative control }}}\right] \times 100
$$

Statistical analysis. The results are expressed as the mean \pm standard deviation. The differences in the means of data were statistically analyzed using one-way analysis of variance (ANOVA), followed by Turkey multiple comparison test. $\mathrm{P}<0.05$ was considered to indicate a statistically significant difference.

\section{Results and Discussion}

\section{Physicochemical characterization of AR liposomes} General appearance. The AR liposomes prepared by each of the TF, REV, and PD methods were light brown, milky suspensions with no precipitation, indicating nanosized vesicles.

Morphology. Fig. 1 shows that liposomes prepared by TF were multilamellar in structure, whereas those prepared by REV and PD were oligolamellar. The differences in morphology and lamellarity of liposomes may be attributed to the different formation mechanisms of vesicles. In the TF method for example, the multilamellar vesicles are formed by hydration of the thin lipid film on the flask wall. Upon addition of the aqueous phase with mechanical agitation, the thin lipid film is swollen and separated from the flask wall into a bilayer, generally forming into liposomes. By contrast, liposomes prepared by the REV method were predominantly uni- or oligo-lamellar vesicles. The mechanism of vesicle formation in this method involves the development of 'inverted micelles' in w/o emulsion, after which slow removal of the organic solvent leads to the transformation of the aforementioned inverted micelles into a viscous gel-like state. Eventually, the gel collapses and transforms into 


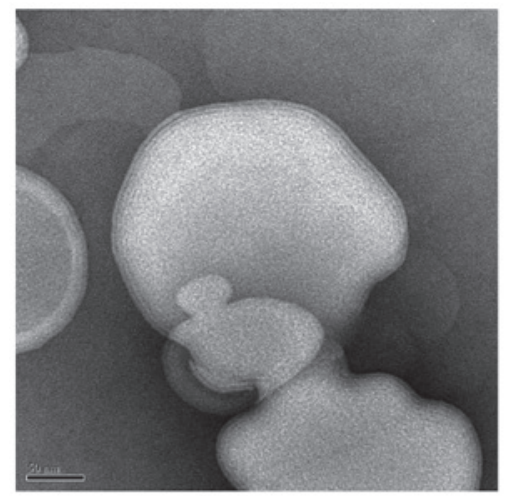

TF

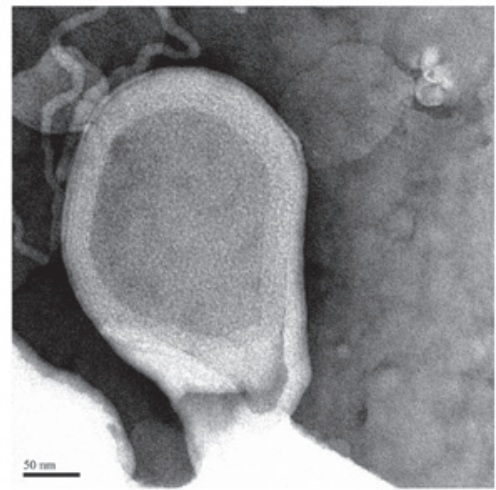

REV

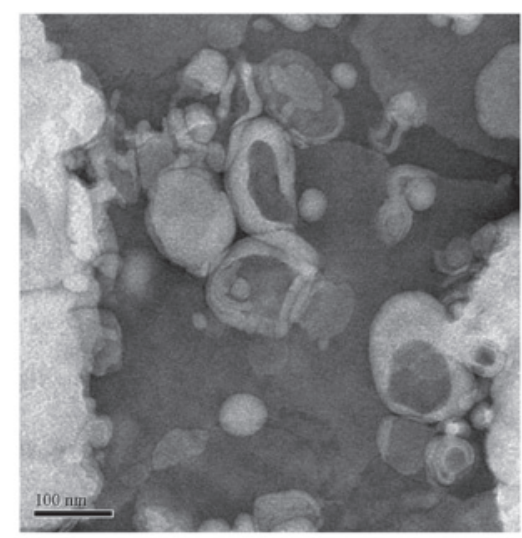

PD

Figure 1. Transmission electron microscope photomicrographs of Asparagus racemosus root extract liposomes prepared by the TF, REV, and PD methods. Magnification, x50,000. TF, thin-film hydration; REV, reverse-phase evaporation; PD, polyol dilution.

a complete bilayer of vesicles $(9,10)$. Conversely, the PD method generally yields relatively large uni- or oligo-lamellar structures. The mechanism of vesicle formation in this method resembles that of the ethanol injection method. Briefly, lipids dissolved in propylene glycol are injected through a fine needle into an excess of aqueous medium to ensure that the propylene glycol is diluted rapidly and the bilayers form instantaneously $(9,11)$.

Particle size. The results presented in Fig. 2 reveal that the mean particle sizes of AR liposomes with various AR to lipid ratios prepared by TF, REV and $\mathrm{PD}$ methods were significantly different from each other $(\mathrm{P}<0.05$; ANOVA), and were in the ranges of 328.1 to $456.6,196.5$ to 239.7 , and 202.4 to $248.3 \mathrm{~nm}$, respectively. The respective polydispersity indexes were $>0.2$, which indicated heterogeneity in the sizes of the obtained AR liposomes prepared by the three different methods. In addition, AR liposomes prepared by the TF method possessed a larger particle size compared with those prepared by REV and PD methods. This may be due to liposomes prepared by the TF method having multilamellar vesicles, while those prepared by the REV and PD methods had oligolamellar vesicles. The different mechanisms of vesicle formation in the three methods was the cause of the observed differences in vesicle type and lamellar structures. However, there appeared to be no association between the mean particle sizes of AR liposomes and the AR to lipid ratios for each method of preparation. These results could be attributed to the water-solubility of AR, which would lead to it being predominantly entrapped in the aqueous compartment rather than in the lipid bilayer of liposomes (12).

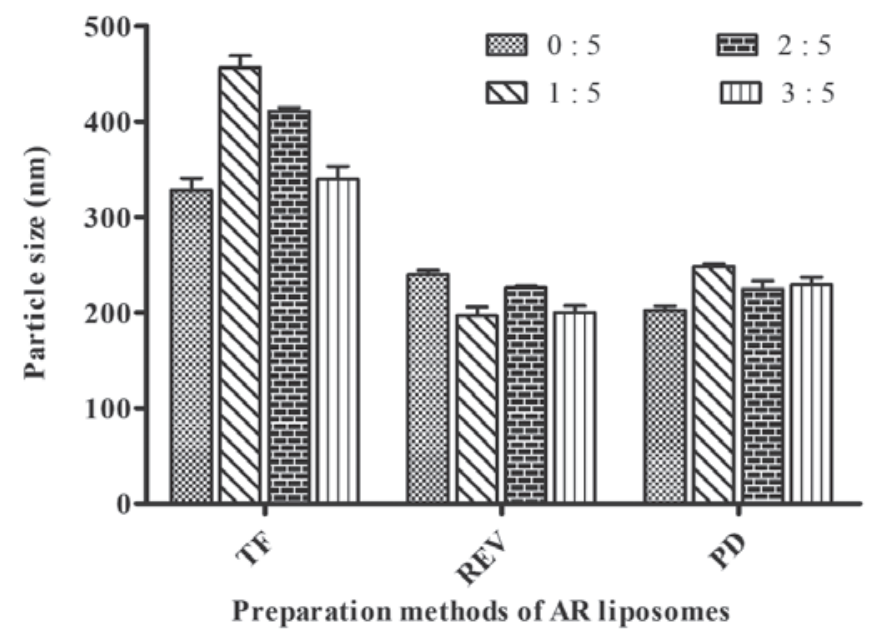

Figure 2. Particle size of AR liposomes prepared by the TF, REV, and PD methods at various AR to lipid weight ratios (0:5, 1:5, 2:5, and 3:5). The data are presented as mean values of three experiments with error bars of standard deviation. AR, A. racemosus root extract; TF, thin-film hydration; REV, reverse-phase evaporation; PD, polyol dilution.

Zeta potential. The mean zeta potentials of AR liposomes with various AR to lipid ratios prepared using the TF, REV, and PD methods were in the ranges of -7.27 to $-11.23,-4.34$ to -12.88 , and -12.08 to $-18.94 \mathrm{mV}$, respectively (Fig. 3). It was found that there were statistically significant differences in the zeta potentials of AR liposomes prepared by these three 


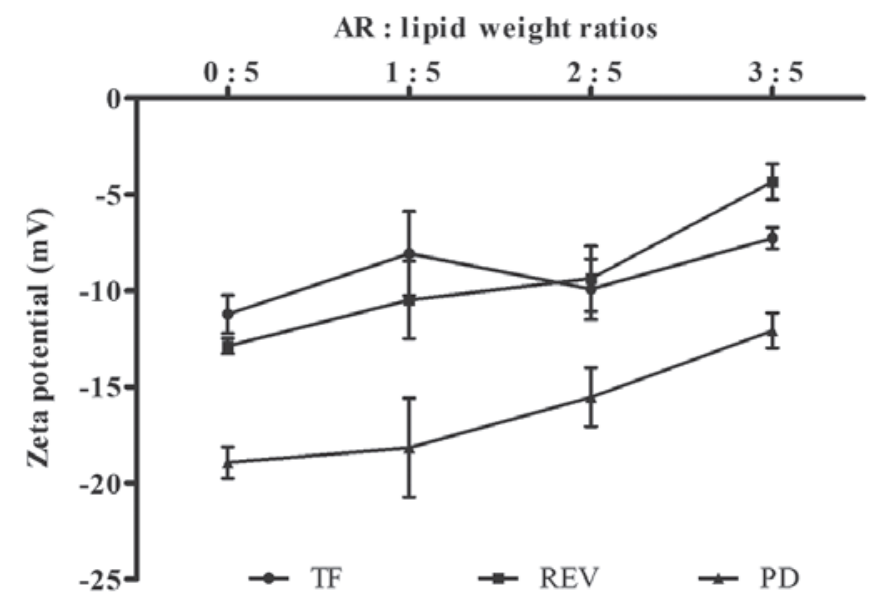

Figure 3. Zeta potential of AR liposomes with AR to lipid ratios of 0:5 1:5, 2:5, and 3:5 prepared by the TF, REV, and PD methods. The data are presented as mean values of three experiments with error bars of standard deviation. AR, Asparagus racemosus root extract; TF, thin-film hydration; REV, reverse-phase evaporation; PD, polyol dilution.

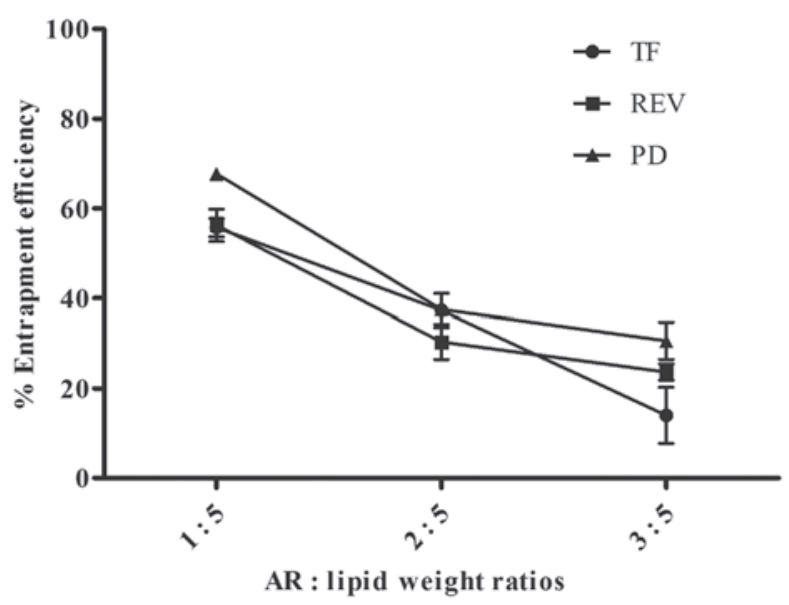

Figure 4. Entrapment efficiency of AR liposomes with AR to lipid ratios of 1:5, 2:5, and 3:5 prepared by the TF, REV, and PD methods. The data are presented as mean values of three experiments with error bars of standard deviation. AR, Asparagus racemosus root extract; TF, thin-film hydration; $\mathrm{REV}$, reverse-phase evaporation; PD, polyol dilution.

methods for each AR to lipid ratio $(\mathrm{P}<0.05$; ANOVA). The zeta potential of AR liposomes prepared by the PD method had a markedly greater negative charge compared with those prepared by TF and PD methods. The aforementioned results reflect the differences in vesicle type and drug localization in liposomes, which is due to the different mechanisms of vesicle formation among the methods of preparation, as previously discussed.

By contrast, the zeta potentials of AR liposomes prepared by each of the three methods were significantly influenced by the AR to lipid ratios ( $\mathrm{P}<0.05$; ANOVA), and had a tendency to increase with increasing amounts of AR. Thus, liposomes with high AR to lipid ratios had a lower negative charge compared with those with low AR to lipid ratios. This observation may be explained on the basis of entrapped AR, the major components of which are saponins having both hydrophilic and hydrophobic parts. The surface-active properties of saponins may exhibit a potential interaction with the phospholipid bilayer of liposomes (13). It has been reported that saponins strongly adsorb onto phospholipid bilayers, and are not readily removed from them, even after purification (14). As a result, the negative charge on the surface of liposomes may be reduced by the entrapped AR.

Entrapment efficiency. The mean values of entrapment efficiency of AR liposomes with various AR to lipid ratios and prepared by TF, REV, and PD methods are shown in Fig. 4, and were in the ranges of 14.02 to $55.71,23.68$ to 56.21 , and 30.62 to $67.68 \%$, respectively. The differences in percentages of entrapment efficiency of AR liposomes prepared by the three methods were statistically significant for the AR to lipid ratios of 1:5 and 3:5 ( $\mathrm{P}<0.05$; ANOVA), however this was not the case for 2:5. These results indicate that the entrapment efficiency is correlated with the method of preparation and the type of liposome. It has been reported that the TF method produces multilamellar vesicles, while the REV and PD methods produce unilamellar and/or oligolamellar vesicles with a large volume of aqueous compartment $(15,16)$. In the present study, AR was soluble in aqueous medium and hence was encapsulated in the aqueous core of the liposomes, similar to other water-soluble compounds. As a result, liposomes prepared by the REV and PD methods would be suitable for entrapment with high volumes of water soluble drug such as AR. However, entrapment efficiency remains dependent on other factors such as bilayer composition and the characteristics of the drug to be encapsulated (17). Furthermore, the present study demonstrated that the percentage of entrapment efficiency significantly decreased with increasing AR to lipid ratio for each method of preparation $(\mathrm{P}<0.05$; ANOVA). The liposomes with an AR to lipid ratio of 1:5 prepared by TF, REV and PD methods possessed the highest percentages of entrapment efficiency $(55.71,56.21$ and $67.68 \%$, respectively).

Cytotoxicity test. The percentage cell viability at various concentrations of AR in aqueous solution (as shown in Fig. 5) was $>90 \%$ within the concentration range of 1 to $1,000 \mu \mathrm{g} / \mathrm{ml}$ compared with the control, indicating no toxic effects on THP-1 cells. Similarly, the percentage cell viability of liposomes without AR (blank liposomes) prepared by various methods was $>90 \%$ at all concentrations within the range from 5 to $500 \mu \mathrm{g} / \mathrm{ml}$, compared with the control, as presented in Fig. 6. The results indicate that the blank liposomes prepared by different methods were not cytotoxic to THP-1 cells. This may be attributed to the bilayer membranes of blank liposomes prepared by different methods being similar to natural cell membranes, which are primarily composed of PC and CHOL.

In vitro anti-inflammatory activity. Fig. 7 shows that unformulated AR possessed substantial anti-inflammatory activity, with $\sim 50 \%$ inhibition of TNF- $\alpha$ at concentrations $<10 \mu \mathrm{g} / \mathrm{ml}$. This activity was comparable to that of the positive control dexamethasone $(1 \mu \mathrm{M})$, which exhibited $64.46 \%$ inhibition. However, no TNF- $\alpha$ inhibition was observed at AR concentrations $>500 \mu \mathrm{g} / \mathrm{ml}$. This may be attributed to the hypothesis that higher concentrations of AR may induce macrophages to produce greater amounts of TNF- $\alpha$. It has been reported that an aqueous extract of AR has able to act as an immunomodulator by increasing the activity of macrophages (18). Furthermore, 


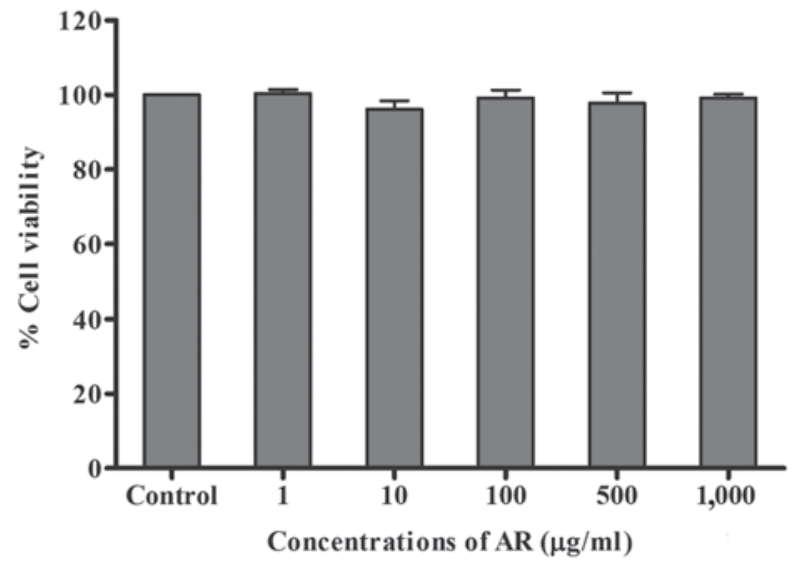

Figure 5. Cytotoxic effect of AR in aqueous solution on THP-1 cells at various concentrations. The data are presented as mean values of three experiments with error bars of standard deviation. AR, Asparagus racemosus root extract.

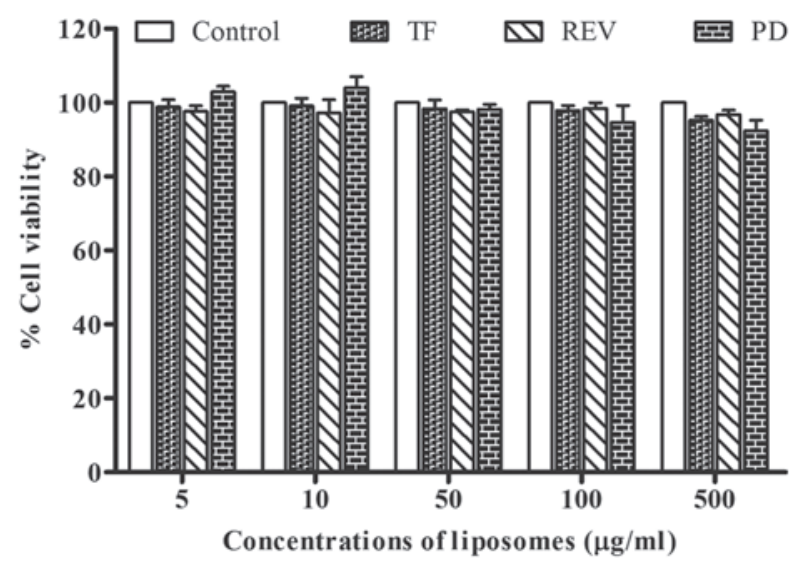

Figure 6. Cytotoxic effect on THP-1 cells of various concentrations of blank liposomes, prepared by the TF, REV, and PD methods. The data are presented as mean values of three experiments with error bars of standard deviation. $\mathrm{TF}$, thin-film hydration; REV, reverse-phase evaporation; $\mathrm{PD}$, polyol dilution.

the levels of TNF- $\alpha$ have been shown to be significantly increased with increasing concentrations of diosgenins, which are phytochemical constituents of AR (19).

The present study also demonstrated that AR liposomes prepared by TF, REV and PD methods exhibited substantial anti-inflammatory activity, particularly at an AR concentration of $0.1 \mu \mathrm{g} / \mathrm{ml}$ (Fig. 8). There were statistically significant differences in the percentage of TNF- $\alpha$ inhibition in AR liposomes prepared by the three different methods using the same AR concentration $(0.1 \mu \mathrm{g} / \mathrm{ml})$, including between TF vs. REV, REV vs. PD and TF vs. $\mathrm{PD}(\mathrm{P}<0.05$; ANOVA). These results suggest that the use of different preparation methods may affect the cellular uptake of liposomes. It is evident that the smaller sized liposomes obtained using the REV and PD methods exhibited greater inhibition of TNF- $\alpha$ compared with those of the larger sized liposomes obtained by use of the TF method. This may be associated with the more efficient liposome-cell membrane interactions of smaller particles compared with those of larger particles $(20,21)$.

Furthermore, AR liposomes at an AR concentration of $1 \mu \mathrm{g} / \mathrm{ml}$ prepared by the PD method displayed the highest

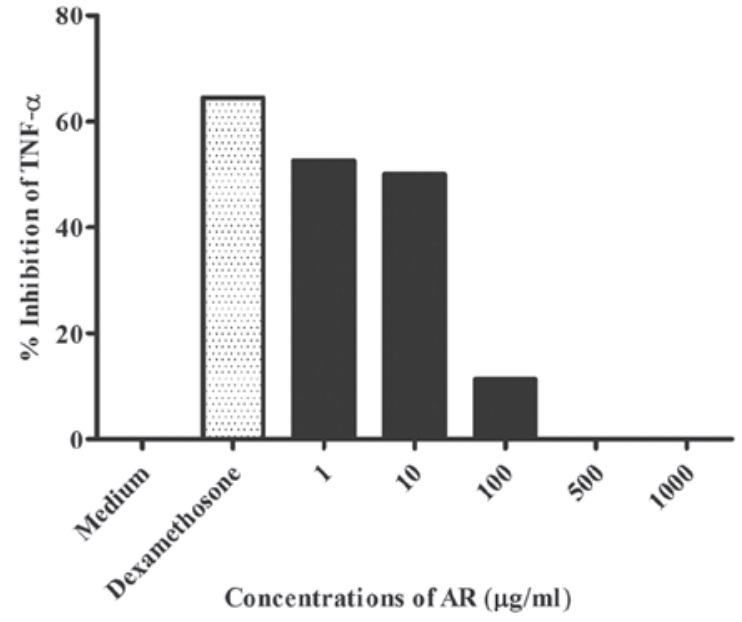

Figure 7. Anti-inflammatory activity of AR $(1-1,000 \mu \mathrm{g} / \mathrm{ml})$ on TNF- $\alpha$ production in lipopolysaccharide-activated THP-1 monocytic cells. The data are presented as mean values of three experiments with error bars of standard deviation. TNF, tumor necrosis factor; AR, Asparagus racemosus root extract.

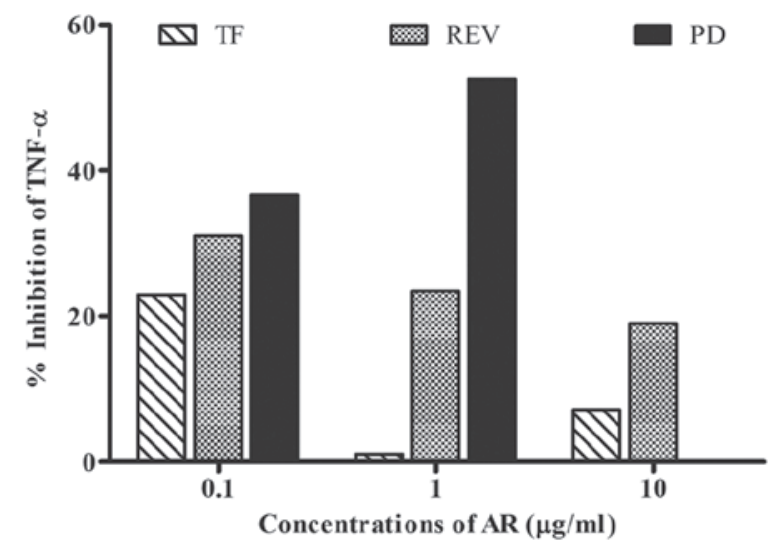

Figure 8. Anti-inflammatory activity of AR, prepared by the TF, REV, and PD methods at AR concentrations of $0.1,1$ and $10 \mu \mathrm{g} / \mathrm{ml}$, on TNF- $\alpha$ production in lipopolysaccharide-activated THP-1 monocytic cells. The data are presented as mean values of two experiments. TNF, tumor necrosis factor; AR, Asparagus racemosus root extract; TF, thin-film hydration; REV, reverse-phase evaporation; $\mathrm{PD}$, polyol dilution.

level of anti-inflammatory activity in the present study, with a percentage inhibition of TNF- $\alpha$ of $52.51 \pm 2.14 \%$, which may be attributed to differences in zeta potentials resulting from different preparation methods. The zeta potentials of AR liposomes prepared by the PD method had a greater negative charges compared with those prepared by TF and REV methods, as illustrated in Fig. 3. It has been postulated that phagocytic cells/macrophages would preferentially uptake negatively charged particles $(22,23)$. Particles containing carboxyl groups with highly negative zeta potentials have been found to be more readily taken up by THP-1 monocytic cells, PMA-differentiated THP-1 cells and macrophages, compared with those containing amine groups with positive zeta potentials (24).

It was also observed that the percentage inhibition of TNF- $\alpha$ tended to decrease with an increasing concentration of AR for those prepared using the TF or REV method (Fig. 8). This result may be attributed to an increased amount 
of liposomal particles being taken into the cells as a result of the increasing AR concentrations. Generally, the uptake of liposomes into cells is a two-step process involving adsorption or binding of liposomes onto the cell surface and subsequent endocytosis or content release $(4,22,23)$. Therefore, increasing the concentration of AR to a certain level in cells may induce macrophages to produce more TNF- $\alpha$, causing the overall percentage of TNF- $\alpha$ inhibition to be reduced. It could suggest that the AR liposomes prepared by the TF, REV and PD methods possessed moderate anti-inflammatory activity at their optimum concentrations.

Conclusions. Use of the TF, REV and PD methods resulted in AR liposomes with different characteristics. Liposomes prepared by the TF method had a multilamellar structure and were of a larger size compared with those prepared by the REV and PD methods, which had oligolamellar structures. However, the mean size of the liposomes obtained using each method had no association with the ratio of AR to lipid. The zeta potential of AR liposomes prepared by the PD method had a greater negative charge compared those prepared by TF and REV methods. The entrapment efficiency significantly decreased with the increasing AR to lipid ratio for each method of preparation, the highest values of which were obtained at an AR to lipid ratio of 1:5. The AR and blank liposomes prepared by these methods had no cytotoxicity to THP-1 cells. AR in addition to AR liposomes exhibited substantial anti-inflammatory activity in terms of percentage inhibition of TNF- $\alpha$, which decreased at higher concentrations of AR.

\section{Acknowledgements}

The authors gratefully acknowledge the financial support provided by the Thai Herbal Nano-Cosmeceuticals Coordinated Research Program, National Nanotechnology Center, National Science and Technology Development Agency, Thailand (grant no. P-00-10647).

\section{References}

1. Bopana N and Saxena S: Asparagus racemosus - ethnopharmacological evaluation and conservation needs. J Ethnopharmacol 110: 1-15, 2007.

2. Alok S, Jain SK, Verma A, Kumar M, Mahor A and Sabharwal M: Plant profile, phytochemistry and pharmacology of Asparagus racemosus (Shatavari): A review. Asian Pac J Trop Dis 3: 242-251, 2013.

3. Amit PC: Mangalesh and RC Roy: Asparagus racemosus (Willd): Biological activities \& its active principles. Indo Glob J Pharm Sci 1: 113-120, 2011.

4. Torchilin VP: Recent advances with liposomes as pharmaceutical carriers. Nat Rev Drug Discov 4: 145-160, 2005.
5. Riaz M: Liposomes preparation methods. Pak J Pharm Sci 9: 65-77, 1996.

6. Sharma A and Sharma US: Liposomes in drug delivery: Progress and limitations. Int J Pharm 154: 123-140, 1997.

7. Ajazuddin and Saraf S: Applications of novel drug delivery system for herbal formulations. Fitoterapia 81: 680-689, 2010.

8. Therdphapiyanak $N$, Jaturanpinyo $M$, Waranuch $\mathrm{N}$, Kongkaneramit L and Sarisuta N: Development and assessment of tyrosinase inhibitory activity of liposomes of Asparagus racemosus extracts. Asian J Pharm Sci 8: 149-160, 2013.

9. New RRC (ed): Introduction, preparation and characterization of liposomes. In: Liposomes: A Practical Approach. 1st edition. Oxford University Press, New York, pp1-161, 1990.

10. Torchillin VP and Weissig V (eds): Liposomes: A Practical Approach. 2nd edition. Oxford University Press, New York, pp1-78, 2003.

11. Betageri GV, Jenkins SA and Parsons DL (eds): Preparation of liposomes. In: Liposome Drug Delivery Systems. Technomic Publishing Co., Basel, pp1-26, 1993.

12. Elmeshad AN, Mortazavi SM and Mozafari MR: Formulation and characterization of nanoliposomal 5-fluorouracil for cancer nanotherapy. J Liposome Res 24: 1-9, 2014.

13. Sapra B, Jain S and Tiwary AK: Effect of Asparagus racemosus extract on transdermal delivery of carvedilol: A mechanistic study. AAPS PharmSciTech 10: 199-210, 2009.

14. Wojciechowski K, Orczyk M, Gutberlet T, Trapp M, Marcinkowski K, Kobiela T and Geue T: Unusual penetration of phospholipid mono- and bilayers by Quillaja bark saponin biosurfactant. Biochim Biophys Acta 1838: 1931-1940, 2014.

15. Szoka F Jr and Papahadjopoulos D: Procedure for preparation of liposomes with large internal aqueous space and high capture by reverse-phase evaporation. Proc Natl Acad Sci USA 75: 4194-4198, 1978.

16. Kikuchi H, Yamauchi H and Hirota S: A polyol dilution method for mass production of liposomes. J Liposome Res 4: 71-91, 1994.

17. Kulkarni SB, Betageri GV and Singh M: Factors affecting microencapsulation of drugs in liposomes. J Microencapsul 12: 229-246, 1995.

18. Veena N, Arora S, Kapila S, Singh RRB, Katara A, Pandey MM, Rastogi $S$ and Rawat AKS: Immunomodulatory and antioxidative potential of milk fortified with Asparagus racemosus (Shatavari). J Med Plants Stud 2: 13-19, 2014.

19. He Z, Tian Y, Zhang X, Bing B, Zhang L, Wang H and Zhao W: Anti-tumour and immunomodulating activities of diosgenin, a naturally occurring steroidal saponin. Nat Prod Res 26: 2243-2246, 2012.

20. Desai MP, Labhasetwar V, Walter E, Levy RJ and Amidon GL: The mechanism of uptake of biodegradable microparticles in Caco-2 cells is size dependent. Pharm Res 14: 1568-1573, 1997

21. Yue H, Wei W, Yue Z, Lv P, Wang L, Ma G and Su Z: Particle size affects the cellular response in macrophages. Eur J Pharm Sci 41: 650-657, 2010.

22. Fröhlich E: The role of surface charge in cellular uptake and cytotoxicity of medical nanoparticles. Int J Nanomedicine 7: 5577-5591, 2012.

23. Mönkkönen J, Valjakka R, Hakasalo M and Urtti A: The effects of liposome surface charge and size on the intracellular delivery of clodronate and gallium in vitro. Int J Pharm 107: 189-97, 1994.

24. Lunov O, Syrovets T, Loos C, Beil J, Delacher M, Tron K, Nienhaus GU, Musyanovych A, Mailänder V, Landfester K and Simmet T: Differential uptake of functionalized polystyrene nanoparticles by human macrophages and a monocytic cell line. ACS Nano 5: 1657-1669, 2011. 